\title{
A mutation which affects both the specificity of PtsG sugar transport and the regulation of ptsG expression by Mlc in Escherichia coli
}

\author{
Jacqueline Plumbridge \\ Tel: +331584150 00.Fax: +331584150 20.e-mail: plumbridge@ibpc.fr
}

Institut de Biologie Physicochimique (UPR9073), 13 rue Pierre et Marie Curie, 75005 Paris, France

\begin{abstract}
Normally glucosamine (GICN) is not a substrate for ElICB ${ }^{G l c}$ of the glucose phosphotransferase system (PTS), encoded by ptsG, but it is transported by the mannose (Man) PTS, encoded by manXYZ. A mutation, umgC, has been described in Escherichia coli which allows a strain mutated in the Man PTS to grow on GIcN. The umgC mutation was mapped to the ptsG region and was proposed to make ptsG expression constitutive. Transcription of ptsG is regulated by the repressor Mlc so that mutations in $\mathrm{m} / \mathrm{c}$ enhance the expression of ptsG. An m/c mutation, however, is not sufficient to allow good growth on GICN, unlike the umgC mutation. The umgC mutation is shown to enhance expression of ptsG even in the absence of any PTS sugar transport, but the increase is greater in the presence of GICN or Man. The umgC mutation also increases expression of the ptsHI and manXYZ operons, which are both regulated by Mlc. The umgC mutation was sequenced and two mutations were found: one, G176D, within the IIC membrane domain and the second, E472K, within the soluble IIB domain of PtsG. The cloned UmgC allele shows the enhanced transport and regulatory characteristics of the chromosomal mutation. Analysis of the two mutations present individually on plasmids shows that the IIC mutation is responsible for both the effect on sugar specificity and regulation.
\end{abstract}

Keywords: Mlc, glucosamine, glucose induction, phosphotransferase system, catabolite repression

\section{INTRODUCTION}

The phosphoenolpyruvate (PEP)-dependent phosphotransferase system (PTS) is the major sugar transport system in bacteria. It consists of a series of proteins which transfer a phosphate from PEP to a sugar as it enters the cell. There are two cytoplasmic components, Enzyme I (EI) and HPr (encoded by the ptsI and ptsH genes) which are common to the transport of most sugars and which pass the phosphate to the sugarspecific membrane components called Enzyme IIs (EIIs). The EIIs are responsible for the phosphorylation of the sugar during its transport across the inner membrane. The EIIs are multidomain proteins where the different

\footnotetext{
Abbreviations: DT, doubling time; Glc, glucose; GlcN, glucosamine; GlcNAc, $N$-acetylglucosamine; Man, mannose; PTS, phosphoenolpyruvate (PEP)-dependent phosphotransferase system; Cm, chloramphenicol; Km, kanamycin; Tc, tetracycline.
}

domains (named IIA, IIB, IIC) are situated on one or several polypeptides (reviewed by Postma et al., 1996). The IIC domain is an intrinsic membrane-spanning protein, whilst the IIA and IIB domains are soluble proteins which in some cases are anchored to the cytoplasmic side of the inner membrane by the IIC domain.

The EIIs can be specific for one sugar or capable of transporting several different sugars. For example, the only known sugar substrate of EII ${ }^{\mathrm{Nag}}$ from Escherichia coli, encoded by nagE, is $\mathrm{N}$-acetylglucosamine (GlcNAc), although EII Nag does transport the antibiotic streptozotocin (Lengeler, 1980). GlcNAc is also transported by the mannose (Man) PTS, EIIABCD Man, encoded by the manXYZ operon. This transporter has a wide substrate specificity, transporting a number of hexoses, including Man, glucose (Glc), fructose and the amino sugars, glucosamine $(\mathrm{GlcN})$ and GlcNAc. On the other hand, the Glc PTS (EIICB ${ }^{\mathrm{Glc}}$ ), encoded by $p t s G$, 
(a)

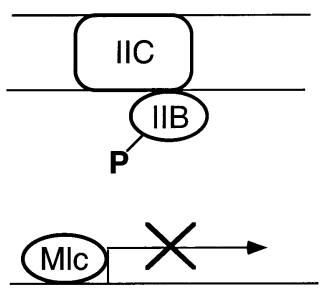

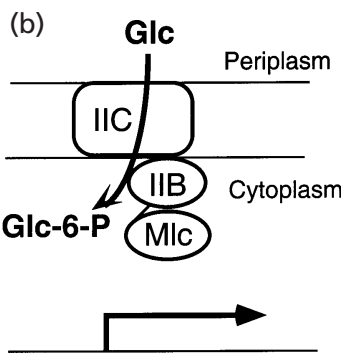

Fig. 1. A model for the regulation of Mlc-controlled promoters by the activity of PtsG. (a) In the absence of Glc, IICB Glc (PtsG) is predominately in its phosphorylated form and Mlc-controlled genes are repressed. (b) Glc is transported across the cytoplasmic membrane via $\mathrm{IIC}^{\mathrm{Gl}}$, it is concomitantly phosphorylated by $\mathrm{IBB}^{\mathrm{Glc}}$ and Mlc-controlled genes are derepressed. One hypothesis to explain the correlation between unphosphorylated $\mathrm{IICB}^{\mathrm{Glc}}$ and derepression of Mlccontrolled genes invokes a physical interaction between Mlc and membrane-associated IICB ${ }^{\mathrm{Gl}}$. It is proposed that the UmgC mutant described in this work partially mimics the conformation normally adopted by $I I C B^{G l c}$ during transport of Glc and hence leads to derepression of Mlc-controlled genes. has a more limited range of substrates; it is the major transporter for Glc but it is also capable of transporting Man and the non-metabolizable Glc analogue $\alpha$-methylglucoside (Curtis \& Epstein, 1975; Kornberg \& JonesMortimer, 1975). PtsG is, however, normally incapable of transporting the amino sugars so that in a wild-type E. coli strain GlcN is only transported by the Man PTS.

A spontaneous mutation was selected which allowed an E. coli manXYZ ( $p t s M)$ mutant strain to grow on GlcN (Jones-Mortimer \& Kornberg, 1980). This mutation, called umgC, mapped to the $p t s G$ loci ( $p t s G$ was previously called $u m g$, uptake of $\alpha$-methylglucoside). It was believed that this mutation rendered the expression of $p t s G$ constitutive and thus capable of transporting GlcN which was not itself capable of inducing $p t s G$ expression, unlike Glc (Jones-Mortimer \& Kornberg, 1980).

It has been shown that expression of $p t s G$ is controlled by the $m l c$-encoded transcription factor. Growth on Glc induces $p t s G$ expression (Erni \& Zanolari, 1986; Korn-

Table 1. E. coli strains

\begin{tabular}{|c|c|c|}
\hline Strain & Genotype* & Source/reference \\
\hline JM2053 & thyA ilvD mglP ptsF ptsG umgC nag kga ptsM pel(am) uhp $p^{\mathrm{c}}$ galP : :Tn10 & $\begin{array}{l}\text { Jones-Mortimer \& Kornberg (1980); } \\
\text { CGSC }\end{array}$ \\
\hline IBPC5321 & thi-1 argG6 argE3 his-4 mtl-1 xyl-5 rpsL $\Delta l a c X 74$ mlc-1 & Plumbridge \& Vimr (1999) \\
\hline IBPC522 & IBPC5321 ptsM8 nagE467 ptsG22 zcf229:: Tn10 & Plumbridge (1990) \\
\hline IBPC703 & IBPC5321 ptsM8 nagE467 umgC zcf229:: Tn10 & $\mathrm{IBPC} 522 \times \mathrm{P} 1(\mathrm{JM} 2053)=\mathrm{GlcN}^{+}$ \\
\hline IBPC704 & IBPC5321 nagE467 ptsG22 zcf229: : Tn10 & IBPC522 $\times$ P1 $\left(\right.$ JM2053) $=\mathrm{GlcN}^{+}$ \\
\hline IBPC707 & IBPC5321 manXYZ::Tn9 & Plumbridge \& Vimr (1999) \\
\hline JM101 & $\mathrm{F}^{\prime}$ traD36 lacl ${ }^{\mathrm{q}} \Delta($ lacZ $) \mathrm{M} 15$ pro $^{+} B^{+} /$supE $\Delta($ lac-proAB $)$ & \\
\hline IBPC1012 & JM101 mlc: :Tc & \\
\hline KD622 & MC4100 $\phi(m a l K-l a c Z) 1113$ (iplacMu50) mlc:: Tn10Km & Decker et al. (1998) \\
\hline CAG12122 & MG1655 zea3125::Tn10Km rph-1 & Singer et al. (1989) \\
\hline JM-G1 & $\mathrm{JM} 101 \lambda \mathrm{RS} / p t s G-l a c Z$ & Plumbridge (1998b) \\
\hline JM-G2 & JM-G1 mlc::Tc & Plumbridge (1998b) \\
\hline JM-G11 & JM-G1 manXYZ:: Tn9 & \\
\hline JM-G19 & JM-G1 manXYZ:: Tn9 mlc:: Tc & \\
\hline JM-G35 & JM-G1 manXYZ:: Tn9 umgC zcf229:: $\operatorname{Tn} 10$ & \\
\hline JM-G55 & JM-G1 manXYZ:: Tn9 umgC zcf229:: Tn10 mlc:: Tn10Km & \\
\hline JM-G88 & JM-G1 umgC zcf229:: Tn10 zea3125::Tn10Km & \\
\hline JM-G62 & $\mathrm{JM}-\mathrm{G} 1 \Delta p t s G:: \mathrm{Cm}$ & \\
\hline JM-G68 & JM-G1 $\Delta p t s G:: \mathrm{Cm} \Delta p t s H I c r r:: \mathrm{Km}$ & \\
\hline JM-G77 & JM-G1 $\Delta p t s G::$ Cm $\Delta p t s H I c r r:: \mathrm{Km} \Delta n a g E B A C D::$ Tc & \\
\hline JM-G96 & JM-G1 $\Delta p t s G::$ Cm ptsM8 zdj225::Tn10 & \\
\hline JM-H1 & JM101 גRS/ptsH-lacZ & Plumbridge (1999) \\
\hline $\mathrm{JM}-\mathrm{H} 2$ & $\mathrm{JM}-\mathrm{H} 1 \mathrm{mlc}: \mathrm{Tc}$ & Plumbridge (1999) \\
\hline JM-H6 & JM-H1 $\operatorname{manXYZ::Tn9}$ & \\
\hline $\mathrm{JM}-\mathrm{H} 30$ & JM-H1 manXYZ:: Tn9 umgC zcf229:: Tn10 & \\
\hline JM-M1 & JM101 $\lambda \mathrm{RS} / \operatorname{manX}-\mathrm{lac} Z$ & Plumbridge (1998a) \\
\hline JM-M2 & JM-M1 mlc::Tc & Plumbridge (1998a) \\
\hline JM-M10 & JM-M1 manXYZ::Tn9 & \\
\hline JM-M13 & JM-M1 manXYZ:: Tn9 umgC zcf229:: Tn10 & \\
\hline
\end{tabular}

* Cm, chloramphenicol; Km, kanamycin; Tc, tetracycline. 


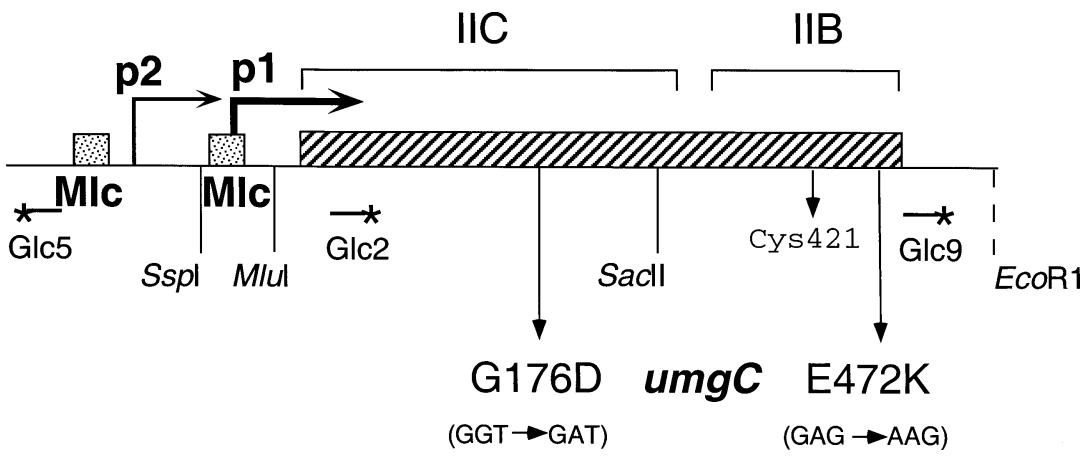

Fig. 2. The ptsG gene and location of the umgC mutation. The relative locations of the IIC and IIB domains are indicated. The two changes found in the umgC strain (G176D and E472K) are shown and the site of phosphorylation, Cys421, within IIB is indicated. pts $G$ is expressed from two promoters, $\mathrm{p} 1$ and $\mathrm{p} 2$, and controlled by Mlc binding to two operators located upstream as shown. The locations of the oligonucleotides Glc2, Glc5 and Glc9 are shown with an asterisk at their $5^{\prime}$ end. Only relevant restriction sites are indicated. The EcoRI site shown with a dotted line is derived from $\mathrm{pBR} 322$ during the cloning. berg \& Reeves, 1972), presumably by relieving Mlc repression (Kimata et al., 1998; Plumbridge, 1998b, 1999). Mlc has also been shown to control the expression of the manXYZ transporter (Plumbridge, 1998a) and the central genes of the PTS, ptsHI (Kim et al., 1999; Plumbridge, 1999; Tanaka et al., 1999). These experiments suggested there was a correlation between the activity of PtsG, in particular the state of phosphorylation of $\mathrm{IIB}^{\mathrm{Glc}}$, and the derepression of Mlccontrolled genes (Fig. 1). In the light of this information, a possible explanation for the effect of the umgC mutation was that it rendered PtsG expression constitutive by affecting Mlc regulation. In this work the $u m g C$ mutation has been characterized and its effect on Mlc regulation of $p t s G$ investigated.

\section{METHODS}

Bacteriological methods and verification of the umgC phenotype. The bacterial strains used are listed in Table 1 . The ptsG-lacZ, ptsH-lacZ and manX-lacZ fusions carried on $\lambda$ bacteriophage have been described previously (Plumbridge, 1998a, b, 1999). The effect of the different mutations on expression of these fusions was tested by introducing the mutations into lysogenic strains by P1 transductions. The umgC mutation was isolated from strain JM2053 (Table 1). A P1 lysate grown on JM2053 was used to transduce the $p t s G 22$ ptsM8 $\left(\mathrm{GlcN}^{-}\right)$strain, IBPC522, selecting for $\mathrm{GlcN}^{+}$. There were two classes of $\mathrm{GlcN}^{+}$bacteria as judged by their colour on MacConkey/GlcN plates and both were $\mathrm{Glc}^{+} \mathrm{Man}^{+}$. The two classes corresponded to those which had become manXYZ $Z^{+}$or $u m g C\left(p t s G^{+}\right)$. Those which had received the umgC mutation (pink-red on MacConkey/GlcN, e.g. IBPC703) remained $\mathrm{GlcN}^{+}$when the $\operatorname{man} X Y Z:$ : Tn9 mutation was introduced, whilst those which had acquired a manXYZ $Z^{+}$ allele (red on MacConkey/GlcN, e.g. IBPC704) lost the ability to grow on GlcN when the manXYZ::Tn9 mutation was introduced. The $u m g C\left(\mathrm{GlcN}^{+}\right)$mutation was shown to be located near to $p t s G$ since it was co-transducible $(5-10 \%)$ with $z c f 229:: \operatorname{Tn} 10$ The work described here shows that the $u m g C$ mutation is in fact an allele of $p t s G$. However, for simplicity and clarity it will be referred to as $u m g C$ to distinguish it from other $p t s G$ mutations. The $z c f 229:$ : Tn10. transposon was used to introduce the $u m g C$ mutation

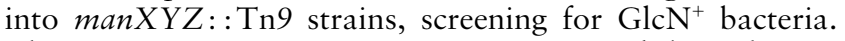
The manXYZ::Tn9 mutation was removed by selecting for zea3125::Tn10Km and screening for $\mathrm{Cm}^{\mathrm{s}}$. The ptsM 8
(manXYZ) mutation was introduced using $z$ dj225::Tn10 (Plumbridge, 1990). Bacteria were grown in minimal MOPS medium at $30^{\circ} \mathrm{C}$ and $\beta$-galactosidase activities were measured as described previously (Miller, 1972; Plumbridge, 1998a).

S1 mapping. Total RNA was prepared from strains JM-G1, JM-G2, JM-G35 and JM-G55 growing on minimal MOPS medium with glycerol or GlcN as carbon source. The probe used was the PCR fragment, Glc5-Glc2 (Fig. 2), labelled at Glc2 to monitor expression from the chromosomal copy of $p t s G$. A second probe covering the $p t s G$-lacZ junction (Glc1Lac22) was used to monitor mRNA from the ptsG-lacZ fusion. Hybridization and S1 analysis were carried out as described previously (Plumbridge, 1998b).

DNA sequencing and cloning of the umgC mutation. PCR fragments covering the $p t s G$ gene (Glc5 to Glc9, Fig. 2) were amplified from chromosomal DNA of the wild-type and the umgC mutated allele (IBPC703), and sequenced using the Thermosequenase Radiolabelled Terminator Cycle Sequencing Kit (Amersham Pharmacia) and a series of internal primers. Plasmid pTZ(PtsG) has been described previously (Plumbridge, 1999). pTZ(UmgC) was made in an analogous way starting from IBPC703. Both plasmids contain DNA corresponding to the $M l u \mathrm{I}$ to Glc9 oligonucleotide (see Fig. 2) so that the $p t s G$ promoter and Mlc operator region is absent and $p t s G$ is expressed from the lac promoter. To separate the G176D mutation from the E472K mutation on pTZ(UmgC), the $\mathrm{N}$-terminal and C-terminal halves of the gene were recloned using the SacII restriction site located near aa 285. The presence of the mutations on the plasmids was verified by sequencing.

\section{RESULTS}

\section{The relative effects of umgC and $m / c$ mutations on growth on GICN and Man}

The umgC mutation of JM2053, conferring growth on GlcN in a manXYZ background, was initially transduced into a strain derived from IBPC5321 (IBPC703). IBPC5321 was subsequently shown to carry a spontaneous mlc $(m l c-1)$ mutation (Plumbridge \& Vimr, 1999). To verify that the $m l_{c-1}$ mutation in IBPC703 was not necessary for the $\mathrm{GlcN}^{+}$phenotype, the $u m g C$ mutation was transduced out of IBPC703 using zcf229:: Tn10 into JM-G11, a manXYZ::Tn9 $\mathrm{mlc}^{+}$ strain derived from JM101, to give JM-G35. Both JM- 
Table 2. Effect of the umgC mutation on growth rates on GlcN and Man, and on expression of ptsG-lacZ

JM101 was lysogenized with $\lambda \mathrm{RS} / p t s G$-lac $Z$ and the mutations indicated were introduced by P1 transduction. All strains are $p t s G^{+}$ except those which carry the $u m g C$ mutation. Bacteria were grown in minimal MOPS medium with $0 \cdot 4 \%$ glycerol or $0 \cdot 2 \%$ GlcN, Man or Glc at $30^{\circ} \mathrm{C}$ as indicated. $\beta$-Galactosidase activities (Miller units, MU) were measured at four points during exponential growth. DTs were calculated by linear regression during the period when the cultures were sampled for $\beta$-galactosidase activity measurements. The data are the means of at least two and mostly four to six independent cultures. NG, No growth.

\begin{tabular}{|c|c|c|c|c|c|c|c|c|c|}
\hline \multirow[t]{2}{*}{ Strain } & \multirow[t]{2}{*}{ Genotype } & \multicolumn{2}{|c|}{ Glycerol } & \multicolumn{2}{|c|}{ GlcN } & \multicolumn{2}{|c|}{ Man } & \multicolumn{2}{|c|}{ Glc } \\
\hline & & $\begin{array}{c}p t s G-l a c Z \\
\quad(\mathrm{MU})\end{array}$ & $\mathrm{DT}(\min )$ & $\begin{array}{c}\text { ptsG-lacZ } \\
\quad(\mathrm{MU})\end{array}$ & $\mathrm{DT}(\min )$ & $\begin{array}{c}\text { ptsG-lac } Z \\
\quad(\mathrm{MU})\end{array}$ & DT (min) & $\begin{array}{c}\text { ptsG-lacZ } \\
\quad(\mathrm{MU})\end{array}$ & $\mathrm{DT}(\min )$ \\
\hline JM-G1 & Wild-type & 8 & 110 & 25 & 150 & 35 & 140 & 59 & 66 \\
\hline JM-G2 & $m l c$ & 140 & 115 & 100 & 100 & 98 & 95 & 59 & 74 \\
\hline JM-G11 & $\operatorname{man} X Y Z$ & 9 & 115 & - & NG & 23 & 300 & 58 & 68 \\
\hline JM-G19 & $\operatorname{manXYZ~mlc~}$ & 130 & 120 & 230 & 600 & 190 & 270 & 63 & 65 \\
\hline JM-G35 & $\operatorname{manXYZ~umgC}$ & 40 & 125 & 160 & 150 & 190 & 160 & 85 & 71 \\
\hline JM-G55 & $\operatorname{manXYZ~umgC~mlc}$ & 150 & 125 & 160 & 155 & 175 & 170 & 82 & 72 \\
\hline JM-G88 & $u m g C$ & 55 & 130 & 125 & 110 & 100 & 95 & 73 & 75 \\
\hline
\end{tabular}

G11 and JM-G35 carry a ptsG-lacZ fusion on a $\lambda$ phage as reporter for $p t s G$ expression. About $5 \%$ of the $\mathrm{Tc}^{\mathrm{R}}$ bacteria became $\mathrm{GlcN}^{+} \mathrm{Man}^{+}$while retaining the manXYZ::Tn9 mutation. This shows that the $u m g C$ mutation is sufficient to confer growth on GlcN to a manXYZ strain in the absence of an mlc mutation. As will be shown, the $u m g C$ mutation is an allele of $p t s G$ but for simplicity it will be called $u m g C$ to distinguish it from $p t s G^{+}$and $p t s G^{-}$alleles.

The capacities of the manXYZ- or umgC-encoded transporters to allow growth on Man and GlcN were investigated by measuring the growth rates of strains using either of these systems in the presence or absence of the mlc mutation (Table 2). Mlc controls the expression of both manXYZ and ptsG. An mlc mutation increases their expression 4- and 20-fold respectively, in low catabolite repression media like glycerol (Plumbridge, 1998a, b, 1999). The effect of the mlc mutation on manXYZ expression is detected by an increase in the growth rate on both GlcN and Man. The introduction of the $m l c$ mutation into a wild-type strain decreases the doubling time (DT) on GlcN from about 150 to $100 \mathrm{~min}$ $\left(30^{\circ} \mathrm{C}\right)$ (Table 2, line 2). A similar decrease in DT is seen with Man. This decrease is almost exclusively due to the effect of the mlc mutation on manXYZ expression since the PtsG-encoded transporter is effectively incapable of allowing growth on GlcN and allows only slow growth on Man. This is shown by the fact that introduction of a manXYZ mutation into a wild-type strain $\left(p t s G^{+} m l^{+}\right)$ eliminates measurable growth on GlcN and increases the DT on Man from about 140 to $300 \mathrm{~min}$ (Table 2, line 3). Subsequent introduction of an $m l c$ mutation into the manXYZ strain has only a very small effect on growth on Man or GlcN. The growth rate on Man is slightly improved and growth on GlcN can now be measured, but with a DT of about $600 \mathrm{~min}$ (Table 2, line 4). This shows that GlcN can be transported, but very in- efficiently, by the PtsG transporter when its expression is increased.

The effect of the introduction of the $m l c$ mutation into a manXYZ strain can be contrasted with the introduction of the $u m g C$ mutation. The latter allows growth rates comparable to the wild-type manXYZ-encoded transporter. The DT of the manXYZ umgC strain on GlcN is about $150 \mathrm{~min}$ compared to $600 \mathrm{~min}$ for the manXYZ $m l c$ strain, using the wild-type PtsG transporter (Table 2 , line 5). Further introduction of an mlc mutation into these manXYZ umgC strains had no appreciable effect on the growth rates. The manXYZ umgC and the manXYZ umgC mlc strains exhibit similar growth rates on all sugars tested. The effect of the $u m g C$ and $m l c$ mutations was also tested during growth on glycerol and Glc. The $u m g C$ mutation produced a slight decrease in the growth rate on glycerol and possibly on Glc, but the changes were relatively small (Table 2 ).

These experiments strongly suggest that constitutive expression of wild-type $p t s G$, as produced by the $m l c$ mutation, is not sufficient to allow PtsG to transport GlcN efficiently and so imply that the $u m g C$ mutation is acting in a different, specific way to permit GlcN and Man uptake and that the $u m g C$ mutation is not just enhancing $p t s G$ expression as expected for a constitutive mutation.

\section{The umgC mutation enhances ptsG expression}

The effect of the umgC mutation on growth rates compared to that of the transcription factor $m l c$ strongly suggests that the $u m g C$ mutation is altering PtsG sugar specificity and thus allowing it to transport GlcN. However, the $u m g C$ mutation does also increase $p t s G$ expression as shown by an effect in trans of the $u m g C$ mutation on the $p t s G-l a c Z$ fusion. Growth of a wild- 
type strain on either GlcN or Man increases ptsG expression three- to fourfold but not so much as growth on Glc (eightfold; Table 2, line 1). These and other PTS sugars are capable of inducing $p t s G$ expression presumably by partially relieving Mlc repression, although the molecular mechanism of the derepression has not been fully elucidated (Plumbridge, 1999). The presence of the $m l c$ mutation derepresses expression in all four media relative to $m l c^{+}$, but the level of $p t s G-l a c Z$ expression observed is different in each medium (Table 2 , line 2). The P1 promoter of the $p t s G$ gene is dependent upon cAMP/CAP and the level of expression in the different sugars is related to the level of catabolite repression generated by growth on the different sugars (Epstein et al., 1975; Hogema et al., 1998; Plumbridge, 1999).

The effect of the umgC mutation on the level of expression of the $p t s G$-lacZ fusion was compared to that of the $m l c$ mutation (Table 2 , lines 2 and 5). Two effects are discernible. Even in a non-PTS medium like glycerol, the umgC mutation causes about a fourfold increase in $p t s G$-lac $Z$ expression. Introduction of an $\mathrm{mlc}$ mutation to the $u m g C$ strain increased expression in glycerol to the same level as in the mlc strain. In GlcN or Man media the $u m g C$ mutation also increases $p t s G$-lacZ expression about five- to sixfold (i.e. at least 20 -fold compared to the basal level of the wild-type strain in glycerol), producing levels of expression even higher than that produced by the mlc mutation in the same medium. Subsequent introduction of the $m l c$ mutation into the $u m g C$ strain had no further effect on expression during growth of the PTS sugars (Table 2, line 6). This can be correlated with the similar growth rates of strains carrying the $u m g C$ and $u m g C m l c$ mutations. Although the presence of the umgC mutation affects $p t s G$ expression in the absence of any PTS substrate, expression is considerably increased in the presence of the UmgC substrates, Man or GlcN. It can also be noted that even in Glc medium the umgC mutation appears to increase $p t s G$ expression somewhat, producing about a $30 \%$ increase in $p t s G$-lac $Z$ expression.

The strong effect of the $u m g C$ mutation during growth on GlcN and Man in the manXYZ background on expression of the $p t s G$-lac $Z$ reporter is reduced by the presence of a manXYZ ${ }^{+}$allele. The presence of a second transporter for Man and GlcN reduces the level of expression of the $p t s G$-lac $Z$ fusion, presumably by reducing the flux through the UmgC transporter. On the other hand the level of expression of $p t s G$-lacZ during growth on glycerol is not affected by the presence of the manXYZ ${ }^{+}$allele.

\section{The umgC mutation enhances ptsG mRNA levels}

The increase in $p t s G$-lacZ expression is paralleled by an increase in chromosomal ptsG mRNA levels as measured by an S1 protection experiment using the Glc5-Glc2 probe (Fig. 2). The umgC mutation produces a small increase during growth on glycerol (Fig. 3, lane

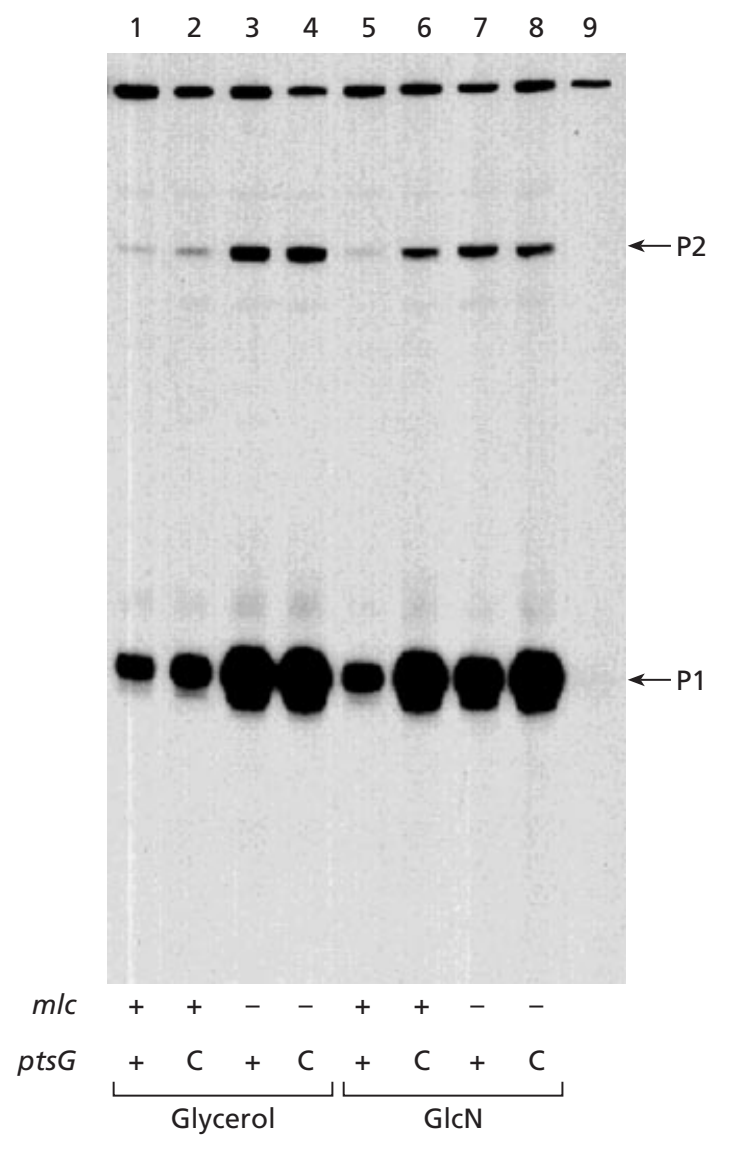

Fig. 3. $\mathrm{S} 1$ analysis of the effect of $u m g C$ mutation on chromosomal ptsG mRNA. The probe used was the GIc5-GIc2 fragment labelled at Glc2. It was hybridized overnight in $40 \mathrm{mM}$ PIPES (pH 6.9), $400 \mathrm{mM} \mathrm{NaCl}, 80 \%$ formamide $\left(52^{\circ} \mathrm{C}\right.$ ) with $25 \mu \mathrm{g}$ total RNA extracted from JM-G1 (lanes 1 and 5), JMG35 (umgC; lanes 2 and 6), JM-G2 (m/c; lanes 3 and 7) and JMG55 (umgC $\mathrm{mlc}$; lanes 4 and 8 ) grown in minimal glycerol medium (lanes 1-4) or minimal GlcN medium (lanes 5-8). C indicates the strain carried the umgC mutation. Lane 9 shows the control with $25 \mu \mathrm{g}$ tRNA. After digestion with S1 nuclease ( 250 units $\mathrm{ml}^{-1}$ in $30 \mathrm{mM}$ sodium acetate, $\mathrm{pH} 4.5,250 \mathrm{mM} \mathrm{NaCl}$, $1 \mathrm{mM} \mathrm{ZnCl}, 5 \%$ glycerol) for $40 \mathrm{~min}$ at $37^{\circ} \mathrm{C}$, S1-resistant hybrids were analysed on a $6 \%$ denaturing acrylamide gel. The major and minor ptsG transcripts, P1 and P2, are indicated.

2) but a considerable increase during growth on GlcN (Fig. 3, lane 6). The mlc mutation in glycerol produces a comparable enhancement in $p t s G$ mRNA (Fig. 3, lanes 3 and 4,) but the mlc mutation in GlcN (Fig. 3, lane 7) seems to produce a somewhat lower level of $p t s G$ mRNA than the umgC mutation, with or without the $m l c$ mutation (Fig. 3, lanes 6 and 8). This correlates with a lower value for the $p t s G$-lac $Z$ fusion in the $m l c$ strain grown on GlcN than for the umgC strain in the same medium (Table 2). The position of the two transcription start sites for $p t s G$, the major P1 transcript and the minor P2 transcript, are not changed by the $u m g C$ mutation and both mRNAs are similarly affected, which is the expected observation if $u m g C$ is altering $\mathrm{Mlc}$ regulation, since Mlc controls both promoters (Plum- 
Table 3. Effect of the umgC mutation on ptsH-lacZ and manX-lacZ expression

JM101 was lysogenized with $\lambda \mathrm{RS} / p t s H$-lacZ or $\lambda \mathrm{RS} / \operatorname{manX}$-lacZ and the mutations indicated were introduced by $\mathrm{P} 1$ transduction. Bacteria were grown in minimal MOPS medium with $0.4 \%$ glycerol (Gly) or $0.2 \%$ GlcN, Man or Glc at $30^{\circ} \mathrm{C}$ as indicated. $\beta$-Galactosidase activities were measured at four points during exponential growth. The data are the means of at least two and mostly three to six independent cultures

\begin{tabular}{|c|c|c|c|c|c|c|c|c|}
\hline \multirow[t]{3}{*}{ Genotype } & \multicolumn{8}{|c|}{$\beta$-Galactosidase activity (Miller units) } \\
\hline & \multicolumn{4}{|c|}{$p t s H-l a c Z$} & \multicolumn{4}{|c|}{$\operatorname{man} X-l a c Z$} \\
\hline & Gly & GlcN & Man & Glc & Gly & GlcN & Man & Glc \\
\hline Wild-type & 420 & 790 & 840 & 1680 & 180 & 350 & 310 & 130 \\
\hline$m l c$ & 1450 & 1730 & 1850 & 1620 & 700 & 540 & 590 & 150 \\
\hline $\operatorname{manXYZ} u m g C$ & 670 & 2020 & 1670 & 1590 & 240 & 930 & 740 & 200 \\
\hline
\end{tabular}

Table 4. Effect of the umgC mutations on plasmids on growth rates and expression of the ptsG-lacZ fusion

JM-G96 ( $\lambda \mathrm{RS} / p t s G$-lacZ, $\Delta p t s G$ ptsM8) was transformed with plasmids carrying pTZ(PtsG) wild-type and the mutant alleles indicated. The UmgC plasmid carries both G176D and E472K. Bacteria were pregrown in minimal MOPS medium with $0 \cdot 4 \%$ glycerol and $0.5 \mathrm{mg}$ ampicillin $\mathrm{ml}^{-1}$, then diluted into medium with $0.2 \%$ carbon source indicated, $0.5 \mathrm{mg}$ ampicillin $\mathrm{ml}^{-1}$ and $10 \mu \mathrm{M}$ IPTG. Exponentially growing bacteria were then rediluted into the same medium and growth rates (DT) and $\beta$-galactosidase activities (Miller units, MU) were measured. Data are the means of at least two independent cultures. There was more variability in both growth rates on Man and GlcN and $\beta$-galactosidase activities in these experiments than when the $p t s G$ mutations were present on the chromosome, presumably due to variations in copy number and IPTG induction. Higher IPTG concentrations did not improve growth rates.

\begin{tabular}{|c|c|c|c|c|c|c|c|c|}
\hline \multirow[t]{2}{*}{ Plasmid ptsG allele } & \multicolumn{2}{|c|}{ Glycerol } & \multicolumn{2}{|c|}{ GlcN } & \multicolumn{2}{|c|}{ Man } & \multicolumn{2}{|c|}{ Glc } \\
\hline & $\begin{array}{c}p t s G-l a c Z \\
(\mathrm{MU})\end{array}$ & $\mathrm{DT}(\min )$ & $\begin{array}{c}p t s G-l a c Z \\
(\mathrm{MU})\end{array}$ & $\mathrm{DT}(\min )$ & $\begin{array}{c}p t s G-l a c Z \\
\quad(\mathrm{MU})\end{array}$ & $\mathrm{DT}(\min )$ & $\begin{array}{c}p t s G-l a c Z \\
(\mathrm{MU})\end{array}$ & $\mathrm{DT}(\min )$ \\
\hline $\mathrm{PtsG}^{+}$ & 8 & 120 & 70 & 300 & 60 & 230 & 60 & 80 \\
\hline UmgC & 30 & 125 & 80 & 110 & 80 & 100 & 62 & 80 \\
\hline G176D & 22 & 120 & 80 & 80 & 70 & 100 & 50 & 75 \\
\hline E472K & 8 & 125 & - & $>500$ & - & $>500$ & 50 & 80 \\
\hline
\end{tabular}

bridge, 1998b). The S1 analysis was repeated using a probe specific for the $p t s G$-lacZ fusion. The pattern of expression was the same as for the chromosomal copy of ptsG (data not shown).

\section{The umgC mutation enhances pts $H$ and $\operatorname{man} X$ expression}

Both the ptsHIcrr and manXYZ operons are controlled by Mlc but the factors of induction, three- to fourfold, are lower than that for $p t s G$ (Plumbridge, 1998a, b, 1999; Table 3). The umgC mutation produces a small increase in expression of both $p t s H$ and $\operatorname{manX}$ during growth on glycerol and larger increases during growth on GlcN and Man, comparable to the presence of the $m l c$ mutation (Table 3). The observation that $u m g C$ has an equivalent effect on $p t s G$, pts $H$ and $\operatorname{manX}$ is in agreement with the idea that the $u m g C$ mutation is affecting their expression via Mlc regulation. Expression of the nagE-encoded GlcNAc transporter is not con- trolled by Mlc and is not significantly affected by the umgC mutation (data not shown).

\section{The umgC allele has two mutations, one in the IIC domain and one in the IIB domain of EIICB ${ }^{\mathrm{Gl}}$}

Sequencing the $p t s G$ chromosomal locus defined by oligonucleotides Glc5 to Glc9, which include $200 \mathrm{bp}$ upstream of the start codon, of the $u m g C$ strain detected two point mutations in the $p t s G$ ORF: G176D in what is predicted to be a periplasmic loop of the IIC domain (Buhr \& Erni, 1993), and E472K near the C-terminal end of the protein (total 477 aa) within the soluble IIB domain (Fig. 2). The pts $G$ locus (from MluI site to oligo Glc9) carrying the $u m g C$ mutation was cloned into a plasmid vector under control of the lac promoter. Plasmids carrying each mutation individually were also constructed. The ability of the plasmids carrying the single and double mutations to allow growth on GlcN and Glc was measured. Both plasmids carrying the 
original double $u m g C$ mutation and the G176D single mutation in the IIC domain allowed growth on GlcN but not the plasmid carrying the E472K IIB mutation (Table 4). In fact, the plasmid carrying the E472K mutation appears to have a negative effect on growth on GlcN and Man. The G176D and double UmgC plasmids also produced an increased expression of ptsG-lacZ during growth on glycerol, showing that it is the IIC mutation which is responsible for both the change in sugar specificity and the increase in $p t s G$ expression.

\section{DISCUSSION}

\section{The umgC mutation affects PtsG sugar transport specificity}

The PtsG protein consists of two domains: an Nterminal membrane-bound EIIC domain predicted to include eight membrane-spanning helices, which is responsible for sugar transport across the cytoplasmic membrane, and a soluble EIIB domain located on the cytoplasmic side of the membrane (Buhr \& Erni, 1993). The EIIB domain contains the Cys421 residue which is phosphorylated by EIIA ${ }^{\text {Glc }}$ and then transfers the phosphate to the transported sugar concomitantly with its passage through the cytoplasmic membrane. Several mutations have been described which affect the sugar specificity of EII ${ }^{\mathrm{Glc}}$ in E. coli. G320V, located in the predicted transmembrane helix VIII (Buhr \& Erni, 1993), allows PtsG to take up mannitol (Begley et al., 1996), five mutations (F37Y, G176D, G281D, I283T and L289Q) have been characterized which allow PtsG to transport ribose (Oh et al., 1999) and one mutation, V12F, has been described which allows the transport of fructose (Kornberg et al., 2000). Ribose is not a PTS sugar and both ribose and fructose were taken up as free sugars by facilitated diffusion rather than by phosphorylation-accompanied transport. Interestingly, the V12F mutation, located in what is predicted to be an $\mathrm{N}$ terminal $\alpha$-helix on the cytoplasmic side of the membrane, was previously isolated in a selection for strains which grew better in a Glc- and oxygen-limited environment (Manché et al., 1999). It shows enhanced Glc transport characteristics as well as the ability to transport fructose (Kornberg et al., 2000; Manché et al., 1999). Two of the ribose-positive mutations were found in regions predicted to be in periplasmic loops of the membrane-spanning region (F37Y and G176D), whilst the three others were clustered in a sequence predicted to form transmembrane helix VII (G281D, I283T and L289Q). In addition, mutations which impair transport but do not affect phosphorylation (Buhr et al., 1992) and mutations which partially uncouple transport from phosphorylation (Ruijter et al., 1992) are found throughout the IIC domain. The wide distribution of these mutations affecting sugar specificity or phosphorylation implies a flexible structure where changes at many locations, both within the membrane and in the connecting loops, affect transport characteristics.

Interestingly, the IIC mutation found in the umgC strain studied here is the same as one of the ribose-positive mutations, G176D (Oh et al., 1999). Moreover, recent experiments, which repeated the selection for a $\mathrm{GlcN}^{+}$ phenotype, yielded several different mutations, including changes in both V12 and G176 (Notley-McRobb \& Ferenci, 2000; Zeppenfeld et al., 2000). As in the case of $\mathrm{V} 12 \mathrm{~F}$, the $u m g C$ mutation also allows uptake of fructose (data not shown), implying that V12F and G176D allow a general reduction in sugar specificity and transportphosphorylation coupling. It should be noted that the umgC mutation does not eliminate all sugar specificity. It does not, for example, allow the uptake of GlcNAc; introduction of the umgC mutation to a manXYZ nagE $\left(\mathrm{GlcNAc}^{-}\right)$strain does not enable the strain to grow on GlcNAc.

\section{The umgC mutation affects Mlc regulation}

Two distinct, but interrelated, phenotypes can be assigned to the $u m g C$ mutation: an enhanced transport capacity for GlcN or Man and an increase in the expression of $p t s G$ and other genes regulated by Mlc. Several pieces of evidence support the hypothesis that the $u m g C$ mutation affects regulation by Mlc: the $u m g C$ mutation produces parallel effects in cis on $p t s G$ mRNA levels and in trans on ptsG-lacZ expression; three different Mlc-controlled operons, ptsG, manXYZ and $p t s H I$, are similarly affected; and the mlc mutation is epistatic to $u m g C$ mutations. During growth on GlcN and Man, when the $u m g C$ mutation has its maximum effect, there is no additional increase in expression when an $m l c$ mutation is introduced, consistent with the idea that the $m l c$ and $u m g C$ mutations are affecting the same regulatory pathway. However, it is clear that the increase in $p t s G$ expression produced by the umgC mutation is not the only reason that growth on GlcN and Man improves since an mlc mutation, which produces increased $p t s G$ expression, does not allow good growth on GlcN or Man. Notice that there is relatively little effect of the $u m g C$ mutation during growth on Glc. This is presumably because the level of catabolite repression generated by Glc dominates the expression level of $p t s G$ and other Mlc-controlled genes, all of which are strongly cAMP/CAP-dependent.

\section{The G176D mutation both causes the change in sugar specificity and the increase in pts $G$ expression}

The analysis of the two mutations found in the umgC strain, G176D and E472K, individually on plasmids show that it is the G176D mutation which is responsible for both phenotypes, i.e. growth on GlcN and increased $p t s G$ expression. This same mutation has already been shown to permit the uptake of ribose. Since several other mutations affecting PtsG sugar specificity map to the IIC domain, it is not surprising that the G176D mutation is responsible for the change in sugar specificity. It is perhaps less expected that the regulatory phenotype, increased expression of Mlc-controlled genes, is also associated with this same mutation. Previous results (Plumbridge, 1999) implicated the soluble IIB domain and the level of PtsG phosphorylation in the regulation of Mlc-controlled genes. Only in the presence of a $p t s G^{+}$ 
allele were Mlc-regulated genes derepressed by growth on PTS sugars or by mutations which interrupted the flow of phosphates from PEP to the sugar.

Growth on Glc reasonably leads to induction by relieving Mlc repression but unlike other classical repressors no low-molecular-mass metabolite has been identified which is capable of displacing Mlc from its DNA targets. One hypothesis to explain the role of PtsG in Mlc induction is that transport of Glc permits an interaction between Mlc and membrane-bound PtsG, thus sequestering Mlc away from its DNA targets (Fig. 1; Plumbridge, 1999). Recent experiments have indeed shown that such an interaction between membranebound PtsG and Mlc exists (S.-J. Lee, W. Boos and J. Plumbridge, unpublished). The predicted location of G176D in a periplasmic loop strongly suggests that there is no direct interaction between G176D and Mlc which is presumably confined to the cytoplasm or the cytoplasmic side of the inner membrane. Instead it would imply that the G176D mutation has a global effect on $\mathrm{IICB}^{\mathrm{Glc}}$ structure which is monitored by $\mathrm{Mlc}$ in the soluble part of the cell. Since the G176D mutation affects regulation in the absence of PTS sugar transport, the mutation might be mimicking an active transport conformation which is able to signal to the IIB domain. There is evidence for such a conformational coupling between the IIC and IIB domains of the mannitolspecific PTS permease (Meijberg et al., 1998). The V12F mutation in the $\mathrm{N}$-terminal cytoplasmic helix of the IIC domain, which shows similar characteristics to G176D (increased $p t s G$ expression and the ability to transport fructose and GlcN), could, however, be in direct contact with Mlc.

The E472K mutation near the $\mathrm{C}$ terminus of the IIB domain appears to have no direct effect on $p t s G$ expression levels. As the G176D mutation in the $u m g C$ strain is sufficient for growth on GlcN, one can wonder what is the origin of the E472K mutation. The presence of a plasmid carrying E472K appears to have a detrimental effect during growth on GlcN or Man compared to the wild-type $p t s G$ allele (Table 4), suggesting that E472K might decrease PtsG activity. If the G176D mutation produces a very strong increase in PtsG transport activity, as has been shown for V12F (Kornberg et al., 2000; Manché et al., 1999), a second mutation reducing this hyperactivity might have arisen. This hypothesis is supported by the observation that when another example of strain JM2053, which had been stored independently for many years, was analysed, it was also found to carry two mutations: G176D and a UAG stop codon at position E472 (L. Notley-McRobb $\& \mathrm{~T}$. Ferenci, personal communication) rather than the AAG lysine found in JM2053 obtained from the CGSC and analysed in this study.

\section{NOTE ADDED IN PROOF}

Biochemical evidence for the model in Fig. 1 comes from the demonstration that Mlc binds to PtsG-containing membranes (Lee et al., 2000; Tanaka et al., 2000).

\section{ACKNOWLEDGEMENTS}

I am very grateful to $\mathrm{H}$. Kornberg, T. Ferenci and K. Jahreis for open discussions and communicating results prior to publication, to A. Kolb, J. Deutscher and W. Boos for many useful discussions, to H. Kornberg and H. Putzer for critical comments on an earlier version of the manuscript and to B. Erni, H. Kornberg, J.-H. Alix and the CGSC for the gift of bacterial strains.

\section{REFERENCES}

Begley, G. S., Warner, K. A., Arents, J. C., Postma, P. W. \& Jacobson, G. R. (1996). Isolation and characterization of a mutation that alters the substrate specificity of the Escherichia coli glucose permease. J Bacteriol 178, 940-942.

Buhr, A. \& Erni, B. (1993). Membrane topology of the glucose transporter of Escherichia coli. J Biol Chem 268, 11599-11603.

Buhr, A., Daniels, G. A. \& Erni, B. (1992). The glucose transporter of Escherichia coli. Mutants with impaired translocation activity that retain phosphorylation activity. J Biol Chem 267, 3847-3851.

Curtis, S. J. \& Epstein, W. (1975). Phosphorylation of D-glucose in Escherichia coli mutants defective in glucose phosphotransferase, mannose phosphotransferase and glucokinase. J Bacteriol 122, 1189-1199.

Decker, K., Plumbridge, J. \& Boos, W. (1998). Negative transcriptional regulation of a positive regulator: the expression of malT, encoding the transcriptional activator of the maltose regulon of Escherichia coli, is negatively controlled by Mlc. Mol Microbiol 27, 381-390.

Epstein, W., Rothman-Denes, L. B. \& Hesse, J. (1975). Adenosine $3^{\prime} 5^{\prime}$-cyclic monophosphate as mediator of catabolite repression in Escherichia coli. Proc Natl Acad Sci U S A 72, 2300-2304.

Erni, B. \& Zanolari, B. (1986). Glucose-permease of the bacterial phosphotransferase system. Gene cloning, overproduction and amino acid sequence of Enzyme $\mathrm{II}^{\mathrm{Gle}}$. J Biol Chem 261, 16396-16403.

Hogema, B. M., Arents, J. C., Bader, R., Eijkemans, K., Yoshida, H., Takahashi, H., Aiba, H. \& Postma, P. W. (1998). Inducer exclusion in Escherichia coli by non-PTS substrates: the role of the PEP to pyruvate ratio in determining the phosphorylation state of Enzyme IIA ${ }^{\mathrm{Glc}}$. Mol Microbiol 30, 487-498.

Jones-Mortimer, M. C. \& Kornberg, H. L. (1980). Amino-sugar transport systems in Escherichia coli K12. J Gen Microbiol 117, 369-376.

Kim, S.-Y., Nam, T.-W., Shin, D., Koo, B.-M., Seok, Y.-J. \& Ryu, S. (1999). Purification of Mlc and analysis of its effects on the pts expression in Escherichia coli. J Biol Chem 274, 25398-25402.

Kimata, K., Inada, T., Tagami, H. \& Aiba, H. (1998). A global repressor $(\mathrm{Mlc})$ is involved in glucose induction of the $p t s G$ gene encoding major glucose transporter in Escherichia coli. Mol Microbiol 29, 1509-1519.

Kornberg, H. \& Jones-Mortimer, M. C. (1975). ptsX: a gene involved in the uptake of glucose and fructose by Escherichia coli. FEBS Lett 51, 1-4.

Kornberg, H. L. \& Reeves, R. E. (1972). Inducible phosphoenolpyruvate-dependent hexose phosphotransferase activities in Escherichia coli. Biochem J 128, 1339-1344.

Kornberg, H. L., Lambourne, L. T. M. \& Sproul, A. A. (2000). Facilitated diffusion of fructose via the phosphoenolpyruvate : glucose phosphotransferase system of Escherichia coli. Proc Natl Acad Sci U S A 97, 1808-1812. 
Lee, S.-J., Boos, W., Bouché, J.-P. \& Plumbridge, J. (2000). Signal transduction between a membrane bound transporter, PtsG, and a soluble transcription factor, Mlc, of Escherichia coli. EMBO J 19 (in press).

Lengeler, J. (1980). Characterisation of mutants of Escherichia coli $\mathrm{K} 12$, selected by resistance to streptozotocin. Mol Gen Genet 179, 49-54.

Manché, K., Nutley-McRobb, L. \& Ferenci, T. (1999). Mutational adaption of Escherichia coli to glucose limitation involves distinct pathways in anaerobic and oxygen-limited environments. Genetics 153, 5-12.

Meijberg, W., Schuurman-Wolters, G. K., Boer, H., Scheek, R. M. \& Robillard, G. T. (1998). The thermal stability and domain interactions of the mannitol permease of Escherichia coli. A differential scanning calorimetry study. J Biol Chem 273, 20785-20794.

Miller, J. H. (1972). Experiments in Molecular Genetics. Cold Spring Harbor, NY: Cold Spring Harbor Laboratory.

Notley-McRobb, L. \& Ferenci, T. (2000). Substrate specificity and signal transduction pathways in the glucose-specific enzyme II $\left(\mathrm{EII}^{\mathrm{Glc}}\right)$ component of Escherichia coli phosphotransferase system. J Bacteriol 182, 4437-4442.

Oh, H., Park, Y. \& Park, C. (1999). A mutated PtsG, the glucose transporter, allows uptake of D-ribose. J Biol Chem 274, 14006-14011.

Plumbridge, J. (1990). Induction of the nag regulon of Escherichia coli by $\mathrm{N}$-acetylglucosamine and glucosamine: role of the cAMPcatabolite activator protein complex in expression of the regulon. J Bacteriol 172, 2728-2735.

Plumbridge, J. (1998a). Control of the expression of the manXYZ operon in Escherichia coli: Mlc is a negative regulator of the mannose PTS. Mol Microbiol 27, 369-381.

Plumbridge, J. (1998b). Expression of $p t s G$, the gene for the major glucose PTS transporter in Escherichia coli, is repressed by Mlc and induced by growth on glucose. Mol Microbiol 29, 1053-1063.
Plumbridge, J. (1999). Expression of the phosphotransferase system (PTS) both mediates and is mediated by Mlc regulation in Escherichia coli. Mol Microbiol 33, 260-273.

Plumbridge, J. \& Vimr, E. (1999). Convergent pathways for utilization of the amino sugars $\mathrm{N}$-acetylglucosamine, $\mathrm{N}$-acetylmannosamine and $\mathrm{N}$-acetylneuraminic acid by Escherichia coli. $J$ Bacteriol 181, 47-54.

Postma, P. W., Lengeler, J. W. \& Jacobson, G. R. (1996). Phosphoenolpyruvate:carbohydrate phosphotransferase systems. In Escherichia coli and Salmonella typhimurium: Cellular and Molecular Biology, pp. 1149-1174. Edited by F. C. Neidhardt, J. L. Ingraham, E. C. C. Lin, K. Brooks Low, B. Magasanik, W. S. Reznikoff, M. Riley, M. Schaechter \& H. E. Umbarger. Washington, DC: American Society for Microbiology.

Ruijter, G. J. G., van Meurs, G., Verwey, M. A., Postma, P. W. \& van Dam, K. (1992). Analysis of mutations that uncouple transport from phosphorylation in Enzyme II $^{\mathrm{Gle}}$ of the Escherichia coli phosphoenolpyruvate-dependent phosphotransferase system. $J$ Bacteriol 174, 2843-2850.

Singer, M., Baker, T. A., Schnitzler, G. \& 7 other authors (1989). A collection of strains containing genetically linked alternating antibiotic resistance elements for genetic mapping of Escherichia coli. Microbiol Rev 53, 1-24.

Tanaka, Y., Kimata, K., Inada, T., Tagami, H. \& Aiba, H. (1999). Negative regulation of the pts operon by Mlc: mechanism underlying glucose induction in Escherichia coli. Genes to Cells 268, 391-399.

Tanaka, Y., Kimata, K. \& Aiba, H. (2000). A novel role of the glucose transporter of Escherichia coli: membrane sequestration of a global repressor Mlc. EMBO J 19 (in press).

Zeppenfeld, T., Larisch, C., Lengeler, J. W. \& Jahreis, K. (2000). Glucose transporter mutants of Escherichia coli K-12 with changes in substrate recognition of the $\mathrm{IICB}^{\mathrm{Glc}}$ and induction behavior of the $p t s G$ gene. J Bacteriol 182, 4443-4452.

Received 9 May 2000; revised 30 June 2000; accepted 13 July 2000. 